\title{
The Evidence of Critical Issues in Transportation Infrastructures of Bangladesh to Introduce Connected and Autonomous Vehicles
}

\author{
Armana Huq 1 , Kamol Debnath Dip ${ }^{2}$, Nadia Binte Mohammad ${ }^{2}$, Nazmus Sakib ${ }^{2}$ \\ ${ }^{1}$ Assistant Professor, Accident Research Institute (ARI) \\ Bangladesh University of Engineering and Technology (BUET), Dhaka 1000, Bangladesh \\ ahuq002@fiu.edu; ashuq@ari.buet.ac.bd \\ ${ }^{2}$ Graduate Research Assistant, Bangladesh University of Engineering and Technology (BUET), Dhaka 1000, Bangladesh, \\ kamoldebnathdip@gmail.com; nadiabmohammad@gmail.com; nazmus046@gmail.com
}

\begin{abstract}
Connected and Autonomous Vehicles (CAVs) have been around for quite some time, and the last decade has seen an upswing of the technology in the transport industry. While some countries have already been laying the groundwork for the successful implementation of CAVs, developing countries like Bangladesh in particular have been lagging in the process. However, Bangladesh is investing in road infrastructure development to comply with Intelligent Transportation Systems (ITS) demand, with the introduction of Electronic Toll Collection (ETC) system establishment in major highways. Introduction of CAVs to any country, of course, depends on market dynamics shaped by people's willingness to pay. The real challenge however lies with the sophisticated connectivity and maintenance demand of CAVs, built upon an infrastructure that requires exacting standards. This study explores such requirements, ranging from traffic signs and road markings, and adequate parking facilities to proper drainage and geometric structure of roadways, following existing guidelines and/or common practices in some of the developed countries. A qualitative evidence synthesis approach is undertaken later to investigate the current infrastructural capability of Bangladesh with respect to these observed benchmarks. In order to assess geometric features, pavement condition, and road markings, a total of 1036 photos were collected and later analyzed from three important intersections of the city of Dhaka. This method was backed up by an extensive literature review of previous studies pertinent to the features that fall under the purview of this study. Key limitations found through the study were assessed in the light of possible measures to overcome them. Finally, recommendations are presented, which albeit need to be investigated of their feasibility and economic viability first. This study intends to provide a baseline of the infrastructure required for introducing the CAVs, which is believed to be applicable for other developing countries with a similar situation.
\end{abstract}

Keywords: Connected and Autonomous Vehicles (CAV), Autonomous Vehicles (AV), Infrastructure Requirements, Bangladesh

\section{Introduction}

Autonomous vehicles (AV) have seen a major leap in technological advancement and implementation in the last few years. Several levels of autonomous features have been experimented in some of the developed countries. As of April 2020, Tesla has incorporated self-driving features in all its vehicles, while other manufacturers like Audi, BMW, Cadillac, Ford, GM, Mercedes-Benz, Nissan, Toyota, Volkswagen, and Volvo - have been testing driverless systems [1]. Full-fledged AV platoons are expected to be roaming the streets by 2040, constituting $30 \%$ of all vehicles [2].

While autonomous vehicle technology has beneficial impacts on the transportation system in its own rights, many preparations need to be undertaken to get a country ready for the introduction of AV into the system. One such particular requirement for the successful implementation of AV technology is infrastructural development. Increased use of AV may impact transport infrastructure in several ways, mainly due to the variation in some parameters including loading levels which might be affected by an improvement in logistics systems and optimization in freight use, and through the change in other factors like operating speed, following distance, and wander across the road width. Further, more frequent use of road networks at night and at more productive levels (platooning of vehicles with shorter following distances) are expected from increased inherent safety systems. This change in variability of parameters provides an opportunity for engineers to construct more optimum designs, and construction and maintenance plans [3].

To analyze the probable effect of AV on a large scale on the rutting performance of pavements, the researcher used a finite element modeling approach [4]. As seen in the study, rutting effect could be exacerbated by reduction in wheel wander and enhancement in lane capacity, which in turn could somewhat be negated by a subsequent increase in traffic speed. 
Therefore, this effect could go either way depending on the practical road and traffic scenario. With that in mind, countries like Japan have mapped the streets in accordance with the implementational requirements of AV technology [5]. On the flip side, little to no research has been conducted in the context of developing countries to assess infrastructural capability to accommodate autonomous vehicles. A survey was conducted in Pakistan where respondents were asked about their perception of roadway safety, but a qualitative assessment was missing [6]. Recently Government of Bangladesh is investing in road infrastructure development to comply with Intelligent Transportation Systems (ITS) demand. Bangladesh has already begun introducing Electronic Toll Collection (ETC) system establishment in major highways [7].

This study aims at addressing the infrastructural scenario of Bangladesh with an eye on the probable introduction of AV fleet in the near future. Roadway parameters including but not limited to pavement structure, lane capacity, traffic signings, roadway markings, and geotechnical characteristics fall under the purview of this study. The findings of this study can be broadcast to the context of other developing countries having the same roadway scenario.

\section{Autonomous and Connected Vehicles}

Connected and Autonomous Vehicles (CAVs) have been around for quite some time, and the last decade has seen an upswing of the technology in the transport industry. While some countries have already been laying the groundwork for the successful implementation of CAVs [8], developing countries like Bangladesh in particular have been lagging in the process. Before proceeding into the heart of the study, necessary background information is reviewed first.

\subsection{Definitions}

Autonomous vehicles (AV) by definition differ from Connected Vehicles (CV). AV is defined as a vehicle which "is designed to be capable of safely completing journeys without the need for a driver in all normally encountered traffic, road and weather conditions" [9]. Connected Vehicles have technological features which enable to communicate with the driver of the vehicle and interact with other vehicular traffic. Three types of communication levels are typically identified as: V2V (vehicle-to-vehicle), V2I (vehicle-to-infrastructure or vice versa, I2V) and V2D (vehicle-to-device or vice versa, D2V) [10]. A device may have many users. To make it more accessible, vehicles to pedestrians' mobile device (V2P) and vehicles to cloud (V2C) technology is included in V2D [10]. Today, Connected and autonomous technologies are blended to give rise to what is known as Connected and Autonomous Vehicles (CAV) [9].

\subsection{Levels of Automation}

Automation is not a binary concept, rather it is achieved through several levels. According to the Society of Automotive Engineers (SAE), six different levels of automation exist. In level 0, human drivers are the only controller of the vehicle. In level 1, some activities are partially automated like acceleration/deceleration. In level 2 automation, several simultaneous activities are partially automated. Level 3 incorporates driver automation where all dynamic driving activities are automated in certain scenarios with the help of human supervision. Level 4 involves high automation with the ability in certain scenarios to cope with humans not intervening. And finally, level 5 has the provisions of full automation where no human intervention is required [10].

\section{Infrastructure Requirements for CAVs and Current Situation in Bangladesh}

At the current stage of Connected Autonomous Vehicle, it requires a certain standard of infrastructure to operate properly. These requirements are discussed here comparing with the current picture of Bangladesh.

\subsection{Traffic Signs and Road Markings}

Road marking is important for the localization of AVs especially for camera-based localization techniques [11]. Errors were seen in determining the location of the vehicle when road marking was missing in straight roads and intersections [11]. The current advanced driving assistant (ADAS) system depends on road markings and some advanced automated systems 
also may depend on markings [12]. The road sign is also important as AV relies on signs for guidance [12]. Without proper maintenance of signs and markings, difficulties in reading information may arise for AVs or CAVs.

From a field visit of 3 intersections in Dhaka city, it was seen that most of the intersections lacked the necessary markings (Table 1). Road links connecting those intersections lacked lane markings and edge marking, also the edge of the pavement was not uniform. The pavement contained potholes and broken edges which reduced the effective lane width. These conditions are very common on many roads of Bangladesh (Fig. 1). Which will create significant difficulties for $\mathrm{AVs} / \mathrm{CAVs}$ moving on these roads.

Table 1: Traffic light, signs, and road marking conditions in 3 visited intersections.

\begin{tabular}{llll}
\hline Condition & Missing & Mostly Faded & Not Used \\
\hline & Stop lines & Pedestrian crosswalk & Traffic signal \\
Rhite line extension & Lane marking & \\
Elements & Lane use arrows & Edge marking & \\
& Stop sign & & \\
& Pedestrian crossing sign & & \\
& Lane merging sign & & \\
& Lane split sign & & \\
\hline
\end{tabular}
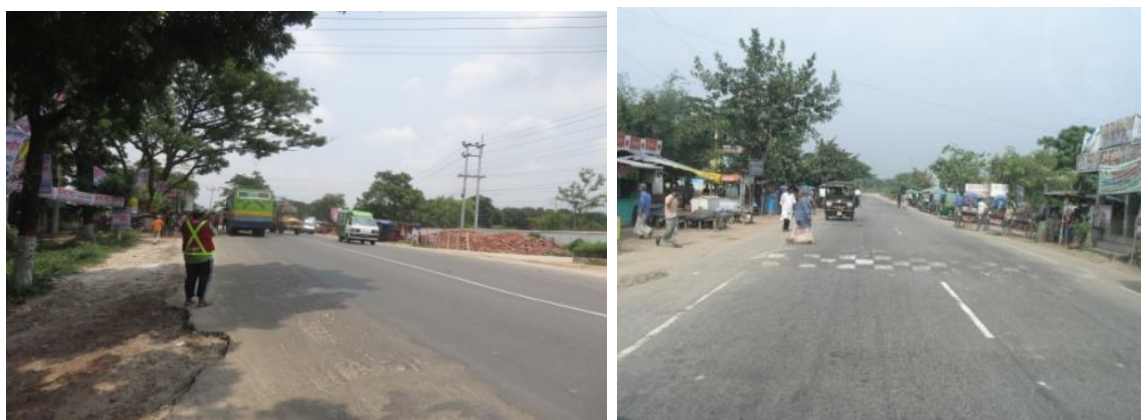

Fig. 1: A highway in Bangladesh with eroded edges (left) and faded markings (right), similar to 3 visited intersections.

\subsection{Incident and Roadwork Communications}

Road incidents, any kinds of road work or construction work in the road will alter the road layout, and CAVs need to depict these differences in real-time, such as lane merging suggestions indicated by temporary signs and cones [13]. AVs need information such as the time of the incident and accurate on-site layout to evaluate the changes from previously mapped data for safe and smooth navigation [13].

Currently, there is no website for real-time information particularly for traffic accidents and road works in Bangladesh. This lacking of real-time information might create a problem for $\mathrm{AVs} / \mathrm{CAVs}$ to navigate in an area where traffic incidents occurred in Bangladesh.

\subsection{Digital Communication}

In excess of vehicle-mounted instruments, CAVs or AVs also need roadside digital infrastructure for various forms of connectivity requirements [14]. Internet connectivity is necessary for substantial amounts of sensors such as loop and magnetic detectors (in-roadway sensors) also cameras, radars and ultrasonic sensors, etc. (over-roadway sensors) [13]. Through I $2 \mathrm{~V}$ communications these sensors help the road authorities in monitoring and allocating traffic resources according to traffic demands [13]. 
No reliable sources have been found that confirm that Bangladesh has implemented these technologies (in-roadway and over-roadway sensors) at a large scale for real-time information. So, it can be assumed that Bangladesh lacks behind in building digital communication infrastructure which is also crucial for AVs.

\subsection{Parking}

Automated Valet Parking (AVP) and High-density parking of automated vehicles can reduce parking demand and also required space up to 50\% [15]. With AVP it is possible to park closer to save space. But it needs a strong GPS signal for operating correctly, without GPS it faces difficulties in navigating a parking space [13]. Addressing this, researchers suggested fitting Bluetooth and near field communications in GPS denied parking space [16].

No parking in Bangladesh is designed for high-density parking, also underground parking lacks a strong GPS signal for the smooth operation of AVP.

\subsection{Service Stations}

Service stations are used for refuelling vehicles sometimes with additional features like shops, maintenance. There is a possibility that all future CAVs will be electric [17]. And also developed countries are trying to increase electric car share on the road [16]. So, service stations with electric chargers for electric vehicles will become a necessity in near future. Even Sweden has already implemented a road with an electric rail that can charge the batteries of cars and trucks driving on it [18].

Most service stations in Bangladesh do not have electric car charging facilities, as electric car ownership is almost non-existent in Bangladesh. There are only 14 electric charging points across the country which is solar-powered [19]. In Bangladesh main battery electric vehicle is Easy Bike [20] which is a three-wheeler and unregistered paratransit [19]. The government is working on an auto industry policy that aims to increase registered vehicles to be powered by "Environmentfriendly electricity" up to $15 \%$ [19].

\subsection{Safe Harbour Area}

To switch automated operations a safe harbour area is necessary for AVs [21]. In developed countries like the UK, the hard shoulder is used as a safe harbour area [13]. Previously safe harbour area referred to emergency refuge area (ERA) which is a $100 \mathrm{~m}$ strip to stop malfunctioned vehicles and located at intervals of $2.5 \mathrm{~km}$. But people misusing ERA reduced its usage [13].

In Bangladesh hard shoulder is provided for roads that have peak traffic flow greater than 800 pcu [22]. But it is intended to use as a running lane when traffic flow increases [22]. So, stopping to switch automated operations in this lane could create a dangerous situation on the highway.

\subsection{Drainage}

As accumulated surface water on pavement can render AVs useless [23], the design and maintenance of drainage infrastructure are important factors in implementing AVs. Improvements in drainage system planning and maintenance (such as culvert, channels and gullies) should be prioritized [10].

Bangladesh faces water logging problems in major cities like Dhaka [24] and Chittagong [25] due to inadequate planning and maintenance. During heavy rainfall, some streets in these cities are flooded with water. Which is not only troublesome for $\mathrm{AVs}$ but also for other conventional vehicles that use them.

\section{Pavement Related Issues}

Bangladesh, being a developing country, vehicle-to-infrastructure (V2I) technologies are critical for the successful implementation of AVs compared to other developed countries. The construction of the pavement and its ancillary features are important for V2I technology. Pavement construction and efficiency are considered to be influenced by four factors: temperature, truck traffic, environment, and the properties of the paving material [26]. 


\subsection{Pavement Structure}

Dr. Audrey Copeland, COO of the National Asphalt Pavement Association gave a presentation in June 2018 on disruptive technologies in which she shared, "Asphalt pavement is well over 90 percent of the U.S. pavement infrastructure. infrastructure. The most cost-effective path to implementing vehicle technologies, such as AVs, is to maintain this infrastructure" [27]. Automated vehicles introduce us to a new traffic trend as they are intended to operate in the middle of of a roadway as per the lane-keeping system (LKS) [28]. Since this process does not permit wheel wandering, this would eventually hasten surface rutting as well as other pavement deteriorations (e.g., potholes, cracking). Wheel wandering was not taken into account in the AASHTO Pavement Design Guide until 1993 [4]. Flexible pavement construction manuals have been revised today to account for wandering. Several experiments have looked at the interdependent relationship between rut depth and wheel wandering and found that little wandering considerably decreases rut depth. According to an analysis when there is no wandering rut depth is $1.19 \mathrm{~mm}$ maximum whereas for $0.26 \mathrm{~m}$ wandering of wheels' accounts for $0.43 \mathrm{~mm}$ rut depth [4]. Hence lies the need of reinforcing certain regions under the CAVs operating route [9].

Bangladesh being a developing country, the road condition is not up to the mark. Also, it is worsening day by day due to poor planning and improper implementation of policies. The existing potholes, edge cracking both in national highway and urban roads (Figure 2) make it difficult to implement CAVs. Also, no such study was done on Bangladesh's roads that provide us with the wandering value.

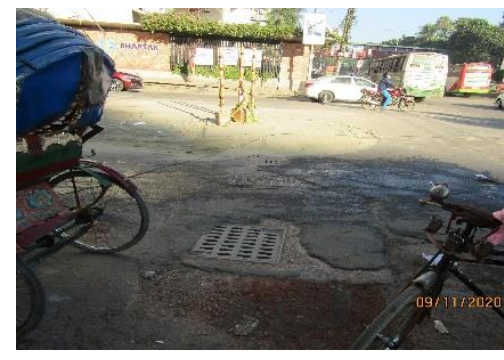

(a)

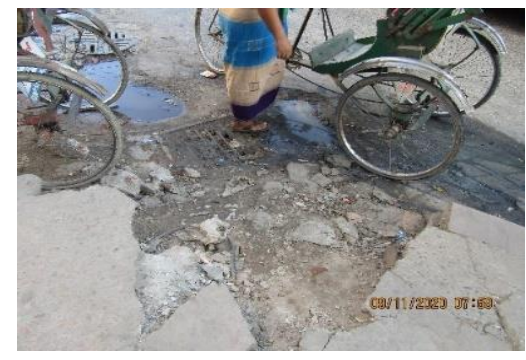

(b)

Fig. 2: An Urban Road in Dhaka City with Potholes (a) and edge Cracking (b).

\subsection{Lane Capacity}

Automated vehicles have the potential to significantly benefit the lane capacity without requiring major infrastructure improvement. However, the typical problem of scarce parking space in the city can be resolved with the introduction of CAVs, which would require half as space as manual vehicles [15]. On the other hand, 100\% increase in capacity can also increase rut depth by $38 \%$ [4].

In Bangladesh, the lane capacity is greatly reduced by the heterogeneity of traffic (Figure 3). Non-motorized and motorized vehicles pass together through the same lane creating a haphazard situation and reducing the effective lane width. Hence providing dedicated lanes for AVs will be very difficult near future.

\subsection{Pavement Material}

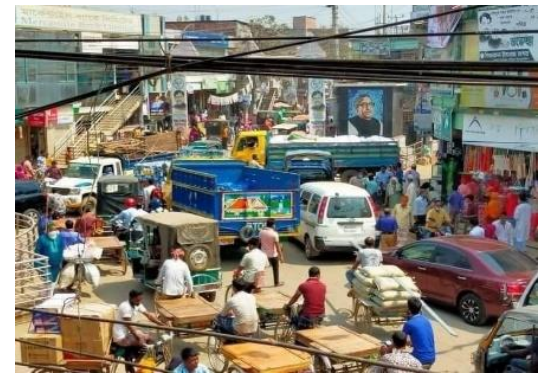

Fig. 3: Traffic Heterogeneity.

Study conducted in The Coastal Highway in Jiangsu Province, at the surface temperature of all $20^{\circ} \mathrm{C}$., tested different variety of pavement combinations in the coastal expressway upper layer which is mainly made of asphalt concrete (AC-13) , stone mastic asphalt (SMA-13), and porous asphalt concrete (PAC-13) mixed materials and result supported SMA-13 to be the best for AV's performance and recommended using SMA-13 in the future intelligent road pavement from the perspective of cost and management [29].

RHD specification for NH is of the 3485 total lengths $98 \%$ is paved and only $2 \%$ is unpaved [30]. However, according to the National Asphalt Pavement Association, 93\% of paved roads are composed of asphalt [31]. Still, to make pavements perpetual, the use of polymer modified binder is a must. Pavements in Bangladesh lack this advancement as well. 


\subsection{Road Surface}

The degree of road surface roughness (as determined by the International Roughness Index, IRI) and friction must be consistent with road markings and signs in full working order. For AV's seamless operation roads will be with sensors to ensure vehicle safety and uninterrupted telecommunications signals.

In Bangladesh our IRI values ranges from 4.00-10.00 according to RHD (Roads and Highways Department) data but due to overloading of vehicles our roads require frequent repair works creating blockage for traffic. High-capacity sensor placement and smooth signaling will be interrupted due to this.

\subsection{Traffic Speed}

To provide us with uninterrupted connectivity, AVs would need higher traffic speeds. However, rut depth in the pavement can be reduced by up to $50 \%$ if the average traffic speed is increased from $10 \mathrm{~km} / \mathrm{h}$ to $50 \mathrm{~km} / \mathrm{h}$ using AVs [4].

According to World Bank statistics, "The average traffic speed in Dhaka has decreased from $21 \mathrm{~km} / \mathrm{h}$ to $7 \mathrm{~km} / \mathrm{h}$ in the last ten years as a result of increasing traffic congestion on roads and unplanned and unregulated development" [32]. The scenarios are almost similar in all other major cities in Bangladesh, with only slight variations. Today, the average traffic speed in Dhaka city is $6.4 \mathrm{kph}$ [33]. However, this average traffic speed could drop to $4.7 \mathrm{kph}$ by 2035-roughly the same as walking, if current vehicle growth trends persist, without additional mass transit incorporation [33]. These are some critical challenges faced in implementing AV in Bangladesh.

\section{Conclusion and Recommendations}

Through a wide-ranging literature review and evidence synthesis, this paper identified the infrastructure barrier in implementing CAVs in answer to the research question "What are the main infrastructure challenges in implementing CAVs in Bangladesh?” Poorly maintained traffic signs, markings, traffic control system, bad road condition, potholes, cracking, lack of service stations for EVs, and inadequate drainage were identified as potential hindrances in implementing CAVs in Bangladesh. To successfully implement this new technology Bangladesh needs to address these infrastructural issues. For this reason, some of the recommendations are discussed below.

- Traffic signs, road markings should be regularly maintained and traffic control system should be properly implemented.

- Different sensors like in-roadway sensors and over-roadway sensors for real-time information should be installed in necessary spots.

- Parking should be designed taking into account the upcoming technologies such as AVs/CAVs.

- The world is shifting toward green electric vehicles. So, the government should implement policies to encourage the use of EVs and create more green energy sources for EVs.

- More extensive studies should be taken to increase the safety of highways in Bangladesh.

- The drainage system of megacities like Dhaka and Chittagong should be redesigned and well maintained to eradicate the water logging problem.

- Incorporating Polymer Modified Binder- SBS, EVA to make bituminous pavements perpetual is necessary. The focus should be given to existing potholes and cracking. Also, heterogeneity of traffic should be systematized as early as possible for operating intelligent signalized intersections.

- More studies should be on Bangladesh's current road condition, road issues, and how to mitigate them to incorporate intelligent transportation with normal traffic.

Further studies can identify more concerning problems with sophisticated data-driven analysis. Also, challenges in other sectors like policy, population, traffic condition, environmental condition, and energy constraints should be explored for Bangladesh. 


\section{References}

[1] Wikipedia contributors. (2021, March 25). Self-driving car [Online]. Available: https://en.wikipedia.org/w/index.php?title=Self-driving_car\&oldid=1014195076

[2] S. A. Bagloee, M. Tavana, M. Asadi and T. Oliver (2016), "Autonomous vehicles: challenges, opportunities, and future implications for transportation policies," Journal of modern transportation, 24(4), 284-303.

[3] J. Wynand and J. W. Maina (2019), "Guidelines for the use of accelerated pavement testing data in autonomous vehicle infrastructure research," Journal of Traffic and Transportation Engineering (English Edition), 6(3), 273-281.

[4] F. Chen, R. Balieu and N. Kringos (2016), "Potential influences on long-term service performance of road infrastructure by automated vehicles," Transportation Research Record, 2550(1), 72-79.

[5] S. Lambert and N. Kareta. (2020). Autonomous vehicles in Japan: A country report. [Online]. Available: https://www.mes-insights.com/autonomous-vehicles-in-japan-a-country-report-a-971640 [Accessed 20 March 2021].

[6] I. Sanaullah, A. Hussain, A. Chaudhry, K. Case and M. Enoch (2017). "Autonomous vehicles in developing countries: A case study on user's view point in Pakistan," in Advances in Human Aspects of Transportation (pp. 561-569). Springer, Cham.

[7] M. Haque, M. Shahrier and A. Huq, "A systematic review of electronic toll collection system," in 1st International Conference on Transportation Research 2020 (ICTR 2020), in press.

[8] UK Intellectual Property Office Informatics Team, Eight Great Technologies Robotics and Autonomous Systems A Patent Overview, Newport, United Kingdom: June 2014.

[9] DfT (2015). The Pathway to Driverless Cars: Summary report and action plan. DfT. Retrieved 20 December 2016 from www.gov.uk/government/uploads/system/uploads/attachment_data/file/401562/pathway-driverless-cars-summary.pdf

[10] C. Johnson (2017). "Readiness of the road network for connected and autonomous vehicles," RAC Foundation: London, UK.

[11] S. Kuutti, S. Fallah, K. Katsaros, M. Dianati, F. Mccullough and A. Mouzakitis, "A survey of the state-of-the-art localization techniques and their potentials for autonomous vehicle applications," in IEEE Internet of Things Journal, vol. 5, no. 2, pp. 829-846, April 2018, doi: 10.1109/JIOT.2018.2812300.

[12] TSC 2017a Future Proofing Infrastructure for Connected and Autonomous Vehicles. Available: https://s3-eu-west1.amazonaws.com/media.ts.catapult/wp-content/uploads/2017/04/25115313/ATS40-Future-Proofing-Infrastructurefor-CAVs.pdf.

[13] Y. Liu, M. Tight, Q. Sun, and R. Kang. "A systematic review: Road infrastructure requirement for connected and autonomous vehicles (CAVs)," in Journal of Physics: Conference Series, vol. 1187, no. 4, p. 042073. IOP Publishing, 2019.

[14] J. Guanetti, Y. Kim and F. Borrelli "Control of connected and automated vehicles: State of the art and future challenges," Annual reviews in control Vol 45, pp. 18-40, 2018.

[15] H. Banzhaf, D. Nienhüser, S. Knoop and J. M. Zöllner, "The future of parking: A survey on automated valet parking with an outlook on high density parking," in 2017 IEEE Intelligent Vehicles Symposium (IV), Los Angeles, CA, USA, 2017, pp. 1827-1834.

[16] UK Autodrive 2018 Paving the Way: Building the Road Infrastructure of the Future for the Connected and Autonomous Vehicles. Available: http://www.ukautodrive.com/downloads/.

[17] P. Godsmark, B. Kirk, V. Gill and B. Flemming "Automated vehicles: The coming of the next disruptive technology"

[18] Boffey D 2018 World's First Electrified Road for Charging Vehicles Opens in Sweden. Available: https://www.theguardian.com/environment/2018/apr/12/worlds-first-electrified-road-for-charging-vehiclesopens-in-sweden.

[19] S. Islam. (2020, December 4). Bangladesh Plans to Attract EV Manufacturers [Online]. Available: https://www.pvmagazine.com/2020/12/04/bangladesh-plans-to-attract-ev-manufacturers/

[20] LighrCastle Analytics Wing. (2020, April 23). Bangladesh's Road to Adoption of Electric Vehicles [Online]. Available: https://databd.co/stories/bangladeshs-road-to-adoption-of-electric-vehicles-10947 
[21] SAE 2018 Taxonomy and Definitions for Terms Related to Driving Automation Systems for On-Road Motor Vehicles. J3016_201806.

[22] Road Design Standards: Standard Design and Costing for Zila, Upazila and Union Roads, Bridges and Culverts, Planning Comission, Government of the People's Republic Bangladesh, 2004. Accessed on: Mar. 19, 2020. [Online]. Available:

https://oldweb.lged.gov.bd/UploadedDocument/UnitPublication/4/14/2004_Road\%20Design\%20Standards.pdf

[23] S. Lawson "Roads that cars can read REPORT III: Tackling the transition to automated vehicle," 2018

[24] Q. A. Mowla and M. S. Islam. "Natural drainage system and water logging in Dhaka: measures to address the problems," Journal of Bangladesh Institute of Planners ISSN 2075 (2013): 9363.

[25] M. A. Ashraf, and M. S. A. Chowdhury. "Drainage planning in the cities of Bangladesh: Case study of drainage and water logging in Chaktai Commercial Area, Chittagong," Journal of Bangladesh Institute of Planners 2 (2009): 49-60.

[26] R.B. Mallick and T. El-Korchi, "Pavement engineering: Principles and practice," CRC Press, Boca Raton, Fla., 2009

[27] G. Fee, n.d. Preparing for autonomous vehicles | Asphalt magazine. [online] Asphaltmagazine.com. Available at: $<\mathrm{http}: / /$ asphaltmagazine.com/autonomous-vehicles/> [Accessed 20 March 2021].

[28] S. L. Vine and J. Polak, “Automated cars: A smooth ride ahead?,” Independent Transport Commission, 2014

[29] Z. Binshuang, C. Jiaying, Z. Runmin and H. Xiaoming, "Skid resistance demands of asphalt pavement during the braking process of autonomous vehicles," in MATEC Web of Conferences (Vol. 275, p. 04002). EDP Sciences, 2019

[30] M. Hasan, "Road classification practice in Bangladesh," in International conference on development in road transportation (DRT 2010), 2010

[31] Ayres, (2016, May 25). Asphalt vs. Concrete: Not a Black and White Choice Online Available: https://www.ayresassociates.com/asphalt-vs-concrete-not-black-white-choice/ [Last Accessed on March 20,2021]

[32] A. Siddique. (2017, July 20). WB: Dhaka's Average Traffic Speed 7 kmph Online Available: https://www.dhakatribune.com/bangladesh/dhaka/2017/07/19/dhaka-average-traffic-speed-7-kmph [ Last Accessed on February 25,2021]

[33] R. Gallagher, "Dhaka's future urban transport: costs and benefits of investment in public and private transport," Copenhagen Consensus Center, 2016. 